\title{
LncRNA loc285194 inhibits tumor growth of laryngeal squamous cell carcinoma cells by downregulating hexokinase 2
}

\author{
YAN GAO $^{1 *}$, ZHONGQIAO WANG $^{1 *}$, JING TONG $^{2}$ and YAN ZHENG $^{1}$ \\ ${ }^{1}$ Department of Otorhinolaryngology, The Second Affiliated Hospital Xinjiang Medical University; \\ ${ }^{2}$ Department of Otorhinolaryngology, The Fifth Affiliated Hospital Xinjiang Medical University, \\ Urumqi, Xinjiang 830000, P.R. China
}

Received August 12, 2018; Accepted March 26, 2019

DOI: $10.3892 / \mathrm{etm} .2019 .7761$

\begin{abstract}
LncRNA loc285194 is a well-characterized tumor suppressor in several types of cancer. To the best of our knowledge, the present study is the first to investigate the involvement of lncRNA loc285194 in laryngeal squamous cell carcinoma (LSCC). The expression of loc285194 and hexokinase 2 (HK-2) mRNA in laryngeal biopsies of patients with LSCC or healthy controls was detected by reverse transcription-quantitative polymerase chain reaction (RT-qPCR). The diagnostic value of loc285194 and HK-2 mRNA for LSCC was evaluated using receiver operating characteristic curve analysis. The correlation between expression of loc285194 and HK-2 mRNA was analyzed using the Pearson correlation coefficient. The association between loc285194 and clinicopathological data of patients with LSCC was analyzed using the Chi-square test. LncRNA loc285194 and HK-2 expression vectors were transfected into human LSCC cell lines and the effects on HK-2 expression, lncRNA loc285194 expression and cell proliferation was detected by western blot, RT-qPCR and CCK-8 assays, respectively. It was observed that loc285194 was upregulated, while HK-2 mRNA was downregulated in patients with LSCC compared with healthy controls. The results demonstrated that the downregulation of loc285194 may be a sensitive diagnostic marker for LSCC. The expression levels of loc285194 and HK-2 mRNA were inversely correlated in patients with LSCC, but not in healthy controls. loc285194 expression was significantly associated with tumor size, but not distant tumor metastasis. Loc285194 overexpression significantly
\end{abstract}

Correspondence to: Dr Yan Zheng, Department of Otorhinolaryngology, The Second Affiliated Hospital Xinjiang Medical University, 38 Nanhu East Road, Shuimogou, Urumqi, Xinjiang 830000, P.R. China

E-mail: qmqtes5@163.com

${ }^{*}$ Contributed equally

Key words: laryngeal squamous cell carcinoma, lncRNA loc285194, hexokinase 2, proliferation inhibited HK-2 expression and LSCC cell proliferation. HK-2 overexpression did not significantly affect Loc285194 expression, but promoted LSCC proliferation and attenuated the inhibitory effects of loc285194 overexpression on LSCC cell proliferation. IncRNA loc285194 may inhibit tumor growth of LSCC cells by downregulating HK-2.

\section{Introduction}

Tumor growth serves pivotal roles in cancer development and progression. Chemical drugs that inhibit tumor cell growth are used in the treatment of cancer (1). Laryngeal squamous cell carcinoma (LSCC) is a rare malignancy, accounting for $\sim 2 \%$ of all cancer cases (2). However, LSCC is considered to be a major cause of cancer-associated mortality due to its aggressive nature and rapid development (3). In spite of the efforts made to prevent and treat LSCC, the survival rate of patients with this disease remains poor (4). Therefore, novel therapeutic approaches with higher efficacies are required to improve treatment outcomes in patients with LSCC. It has been reported that tumor size is associated with the prognosis of patients with LSCC (5). Therefore, the identification of factors that can regulate tumor growth in patients with LSCC may provide guidance for the treatment of LSCC.

Hexokinase 2 (HK-2) is an enzyme in the human body that can phosphorylate glucose to produce glucose-6-phosphate, which is typically the initial step in glucose metabolism (6). The overexpression of HK-2 is frequently observed in the progression of different types of cancer, and the upregulated expression of HK-2 in certain types of cancer accelerates cell growth and promotes cancer progression (7-9). Thus, the inhibition of HK-2 expression is considered to be a promising therapeutic target for cancer treatment (10). HK-2 achieves its biological roles through its interactions with different cellular factors, including long (>200 nucleotides) non-coding RNAs (lncRNAs), which are a heterogeneous group of RNA transcripts with pivotal roles in human diseases due to their regulatory roles in gene expression (11). LncRNA loc285194 has been reported to have tumor suppressive functions in certain types of malignancies (12), while its involvement in LSCC remains unclear. Our preliminary data of microarray revealed an association between HK-2 and loc285194 (data not shown). The present study aimed to assess the involvement 
of lncRNA loc285194 in the pathogenesis of LSCC via its interaction with HK-2.

\section{Materials and methods}

Subjects and specimens. The current study included 66 patients with LSCC, who were diagnosed at the Affiliated Second Hospital Xinjiang Medical University between January 2015 and March 2017. The inclusion criteria were as follows: i) Patients diagnosed through laryngeal biopsies; ii) patients received treatment for the first time; iii) patients willing to join the study. The exclusion criteria were as follows: i) Patients complicated with other malignancies (102 patients were excluded); ii) patients who were treated prior to admission (12 cases were excluded); iii) patients with chronic diseases (45 patients were excluded); iv) blood relatives ( 2 patients were excluded). Among those patients, 10 cases were in American Joint Committee on Cancer stage II, 12 in stage III, 9 in stage IVA, 12 in stage IVB and 23 in stage IVC. The patients included 36 males and 30 females with ages ranging between 24 and 66 years, and a mean age of $44.5 \pm 6.6$ years. During the same time period, 92 people received laryngeal biopsies to detect suspected laryngeal lesions and laryngeal lesions were excluded in 39 cases. Among these 39 cases, laryngeal biopsies were obtained from 28 cases to match the age and sex distributions of patient group and to serve as control group. The present study was approved by the Ethics Committee of The Affiliated Second Hospital Xinjiang Medical University. All participants signed informed consent. Laryngeal biopsies were obtained from the specimen library of the Affiliated Second Hospital Xinjiang Medical University. All specimens were stored in liquid nitrogen $\left(-195.79^{\circ} \mathrm{C}\right)$ prior to use.

Reverse transcription-quantitative polymerase chain reaction ( $R T-q P C R)$. TRIzol reagent (Invitrogen; Thermo Fisher Scientific, Inc., Waltham, MA, USA) was used to extract total RNA. All operations were performed in accordance with manufacturer's protocol. cDNA was synthesized through reverse transcription using SuperScript III Reverse Transcriptase (Thermo Fisher Scientific, Inc.) under the following conditions: $50^{\circ} \mathrm{C}$ for $30 \mathrm{~min}$ and $75^{\circ} \mathrm{C}$ for $15 \mathrm{~min}$. qPCR was performed using SYBR ${ }^{\circledR}$ Green Real-Time PCR Master Mix (Thermo Fisher Scientific, Inc.) with following primers: lncRNA loc285194 forward, 5'-TGTGCCTGTTTG ACCTCTGA-3' and reverse, 5'-AGGAAGGATAAAAGACCG ACCA-3'; HK-2 mRNA forward, 5'-AAGGCTTCAAGGCAT CTG-3' and reverse, 5'-CCACAGGTCATCATAGTTCC-3'; $\beta$-actin forward, 5'-GACCTCTATGCCAACACAGT-3' and reverse, 5'-AGTACTTGCGCTCAGGAGGA-3'. The qPCR reaction conditions were as follows: $50 \mathrm{sec}$ at $95^{\circ} \mathrm{C}$, followed by 40 cycles of $20 \mathrm{sec}$ at $95^{\circ} \mathrm{C}$ and $40 \mathrm{sec}$ at $59^{\circ} \mathrm{C}$. The $2^{-\Delta \Delta \mathrm{Cq}}$ method was used for data normalization (13).

Cell lines, cell culture and cell transfection. Human LSCC cell line UM-SCC-17A (EMD Millipore, Billerica, MA, USA) and normal larynx cell line HuLa-PC (American Type Culture Collection, Manassas, VA, USA) were used in this study. Cells of each cell line were cultured with DMEM containing 10\% fetal bovine serum (FBS; Sangon Biotech Co., Ltd. Shanghai, China) at $37^{\circ} \mathrm{C}$ with $5 \% \mathrm{CO}_{2}$. Full-length loc285194 and $\mathrm{HK}-2$
cDNAs were amplified through PCR using primers with NheI cutting site at the 5' end. Loc285194 and HK-2 cDNAs were inserted into pIRSE2 vector (Clontech Laboratories, Inc., Mountainview, CA, USA) to construct loc285194 and HK-2 expression vectors. Loc285194 and HK-2 expression vectors were transfected into $5 \times 10^{5}$ cells at a dose of $10 \mathrm{nM}$ using Lipofectamine 2000 reagent (Thermo Fisher Scientific, Inc.). Cells without transfection were used as control cells. Cells transfected with empty vector were used as negative control cells. The expression of loc285194 and HK-2 was confirmed by RT-qPCR $12 \mathrm{~h}$ after transfection. Subsequent experiments were performed only in cases where an overexpression rate of $>200 \%$ compared with control cells and negative control cells was reached.

Cell Counting Kit-8 (CCK-8) assay. Cell proliferation was detected using a CCK-8 assay. Briefly, cell suspensions (1\% FBS) with a cell density of $5 \times 10^{4}$ cells $/ \mathrm{ml}$ were prepared. Subsequently, $5 \times 10^{3}$ cells in $0.1 \mathrm{ml}$ cell suspension were added into each well of a 96-well plate. Cells were cultured at $37^{\circ} \mathrm{C}$ in a $5 \% \mathrm{CO}_{2}$ incubator, followed by addition of $10 \mu \mathrm{lCCK}-8$ solution (Sigma-Aldrich; Merck KGaA, Darmstadt, Germany) 24, 48, 72 and $96 \mathrm{~h}$ later. Cells were cultured for additional $4 \mathrm{~h}$ and Fisherbrand ${ }^{\mathrm{TM}}$ accuSkan $^{\mathrm{TM}}$ GO UV/Vis microplate spectrophotometer (Thermo Fisher Scientific, Inc.) was used to measure the optical density values at $450 \mathrm{nM}$.

Western blot analysis. RIPA solution (Thermo Fisher Scientific, Inc.) was mixed with cultured cells to extract total protein. Subsequently, a bicinchoninic acid assay was performed to measure protein concentration. Protein samples were denatured and subjected to $10 \%$ SDS-PAGE gel electrophoresis with $45 \mu \mathrm{g}$ protein/lane. Subsequently, PVDF membranes were blocked in 5\% skimmed milk at room temperature for $1 \mathrm{~h}$, followed by incubation with rabbit anti-human primary antibodies against HK-2 (1:1,200; cat. no. ab37593; Abcam, Cambridge, UK) and GAPDH (1:2,000; cat. no. ab8245; Abcam) at $4^{\circ} \mathrm{C}$ overnight. The following day, a goat anti-rabbit IgG-horseradish peroxidase secondary antibody $(1: 1,000$; cat. no. MBS435036; MyBioSource, San Diego, CA, USA) was used to further incubate with membranes at $25^{\circ} \mathrm{C}$ for $2 \mathrm{~h}$. Then, ECL (Sigma-Aldrich, Merck KGaA) was added to develop the signals. Signals were normalized using ImageJ v1.46 software (National Institutes of Health, Bethesda, MD, USA).

Statistical analysis. Data analyses were performed using GraphPad Prism 6 software (GraphPad Software, Inc., La Jolla, CA, USA). Gene expression and cell proliferation data are presented as the mean \pm standard deviation. One-way analysis of variance followed by the least significant difference test among multiple groups or unpaired t-test between two groups was used. Associations between loc285194 expression and clinicopathological data of LSCC patients were analyzed using the Chi-square test. Correlations between expression of loc285194 and HK-2 mRNA were analyzed using the Pearson correlation coefficient. The diagnostic value of loc285194 and HK-2 mRNA for LSCC was evaluated using receiver operating characteristic curve analysis (ROC). Origin Pro 8.0 (OriginLab, Northampton, MA, USA) was used to calculate statistical power. Statistical 
A

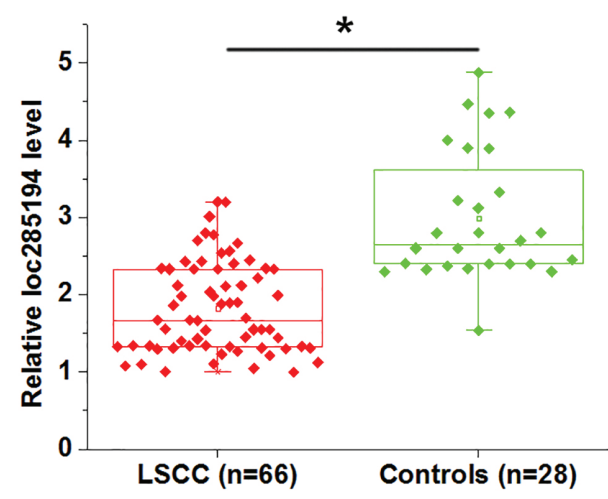

B

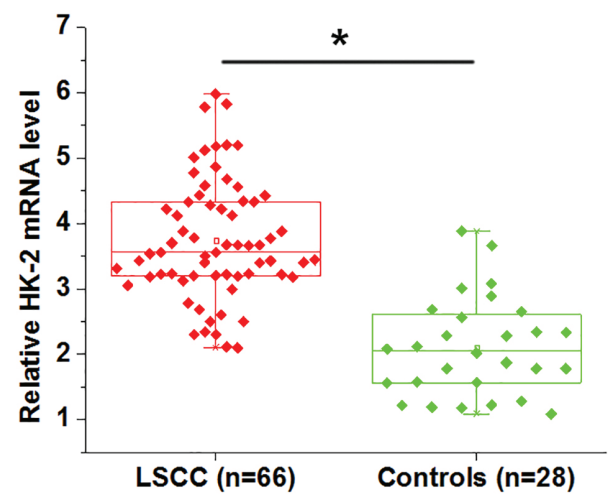

Figure 1. Loc285194 is downregulated and HK-2 mRNA is upregulated in patients with LSCC compared with in healthy controls. Comparison of loc285194 and HK-2 mRNA expression revealed (A) significantly downregulated loc285194 expression and (B) significantly upregulated HK-2 mRNA expression in patients with LSCC compared with in healthy controls. " $\mathrm{P}<0.05$. LSCC, laryngeal squamous cell carcinoma; HK-2, hexokinase 2.
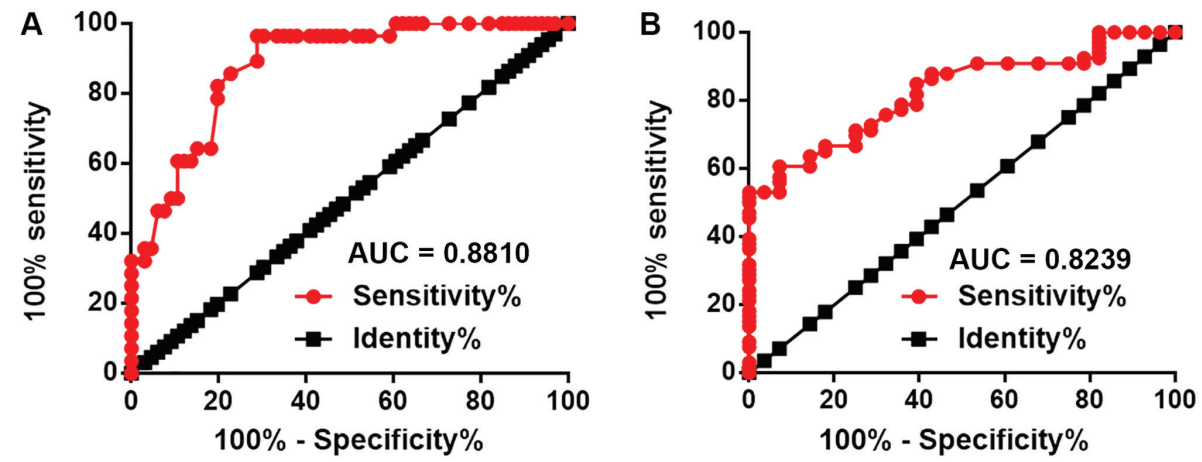

Figure 2. Loc285194 and HK-2 mRNA are potential diagnostic biomarkers for LSCC. Receiver operating characteristic curve analysis revealed that (A) downregulation of loc285194 and (B) upregulation of HK-2 mRNA effectively distinguished patients with LSCC from healthy controls. LSCC, laryngeal squamous cell carcinoma; HK-2, hexokinase 2; AUC, area under the curve.

power $>90 \%$ was reached in all cases. All data provided sufficient statistical power. $\mathrm{P}<0.05$ was considered to indicate a statistically significant difference.

\section{Results}

Loc285194 is downregulated and HK-2 mRNA is upregulated in patients with LSCC compared with healthy controls. The expression of loc285194 and HK-2 mRNA in laryngeal biopsies of patients with LSCC or healthy controls was detected using RT-qPCR. As shown in Fig. 1A, loc285194 expression was significantly downregulated in patients with LSCC compared with that in healthy controls $(\mathrm{P}<0.05)$. In contrast, HK-2 mRNA was significantly upregulated in LSCC patients compared with that in healthy controls (Fig. 1B; $\mathrm{P}<0.05$ ).

Loc285194 and HK-2 mRNA are potential diagnostic biomarkers for LSCC. ROC curve analysis was performed to evaluate the diagnostic values of loc285194 and HK-2 mRNA for LSCC (Fig. 2). For loc285194, the area under the curve (AUC) was 0.8810 [standard error (SE), 0.03499; 95\% confidence interval (CI), 0.8124-0.9405; $\mathrm{P}<0.0001]$. For HK-2 mRNA, the AUC was 0.8239 (SE, 0.04211; 95\% CI, 0.7413-0.9064; $\mathrm{P}<0.0001)$. loc285194 and HK-2 mRNA expression levels are inversely correlated in patients with LSCC, but not in healthy controls. The correlation between the expression levels of loc285194 and HK-2 mRNA was analyzed using the Pearson correlation coefficient. A significantly inverse correlation was identified between the expression levels of loc285194 and HK-2 mRNA in patients with LSCC (Fig. 3A). In contrast, no significant correlation between loc285194 and HK-2 mRNA expression levels was observed in healthy controls (Fig. 3B).

Expression of loc285194 is significantly associated with tumor size, but not distant tumor metastasis. Patients were divided into high and low expression groups according to the median expression level of loc285194 (1.72). The association between loc285194 and clinicopathological data of patients with LSCC was analyzed using the Chi-square test. As shown in Table I, loc285194 expression was not significantly associated with the age, sex, smoking and drinking habits of patients as well as the presence of tumor metastasis. In contrast, a significant association was observed between loc285194 expression and primary tumor size.

Loc285194 is an upstream activator of HK-2 in LSCC cells. Cells with transient loc285194 and HK-2 were produced, and the expression of loc285194 and HK-2 was detected by 
Table I. Associations between loc285194 expression and clinicopathological data of LSCC patients.

\begin{tabular}{|c|c|c|c|c|c|}
\hline \multirow[b]{2}{*}{ Characteristic } & \multirow[b]{2}{*}{ Cases } & \multicolumn{2}{|c|}{ Expression } & \multirow[b]{2}{*}{ Chi-square } & \multirow[b]{2}{*}{ P-value } \\
\hline & & High & Low & & \\
\hline \multicolumn{6}{|l|}{ Sex } \\
\hline Male & 36 & 16 & 20 & 0.98 & 0.32 \\
\hline Female & 30 & 17 & 13 & & \\
\hline \multicolumn{6}{|l|}{ Age, years } \\
\hline$>45$ & 32 & 14 & 18 & 0.97 & 0.32 \\
\hline$\leq 45$ & 34 & 19 & 15 & & \\
\hline \multicolumn{6}{|l|}{ Smoking } \\
\hline Yes & 28 & 12 & 16 & 0.99 & 0.32 \\
\hline No & 38 & 21 & 17 & & \\
\hline \multicolumn{6}{|l|}{ Drinking } \\
\hline Yes & 33 & 15 & 18 & 0.55 & 0.46 \\
\hline No & 33 & 18 & 15 & & \\
\hline \multicolumn{6}{|l|}{ Tumor size, $\mathrm{cm}$} \\
\hline$\geq 6$ & 19 & 5 & 14 & 6.02 & 0.049 \\
\hline $3-6$ & 23 & 14 & 9 & & \\
\hline$\leq 3$ & 24 & 14 & 10 & & \\
\hline \multicolumn{6}{|c|}{ Distant metastasis } \\
\hline Yes & 43 & 19 & 24 & 1.67 & 0.196 \\
\hline No & 23 & 14 & 9 & & \\
\hline
\end{tabular}

LSCC, laryngeal squamous cell carcinoma.
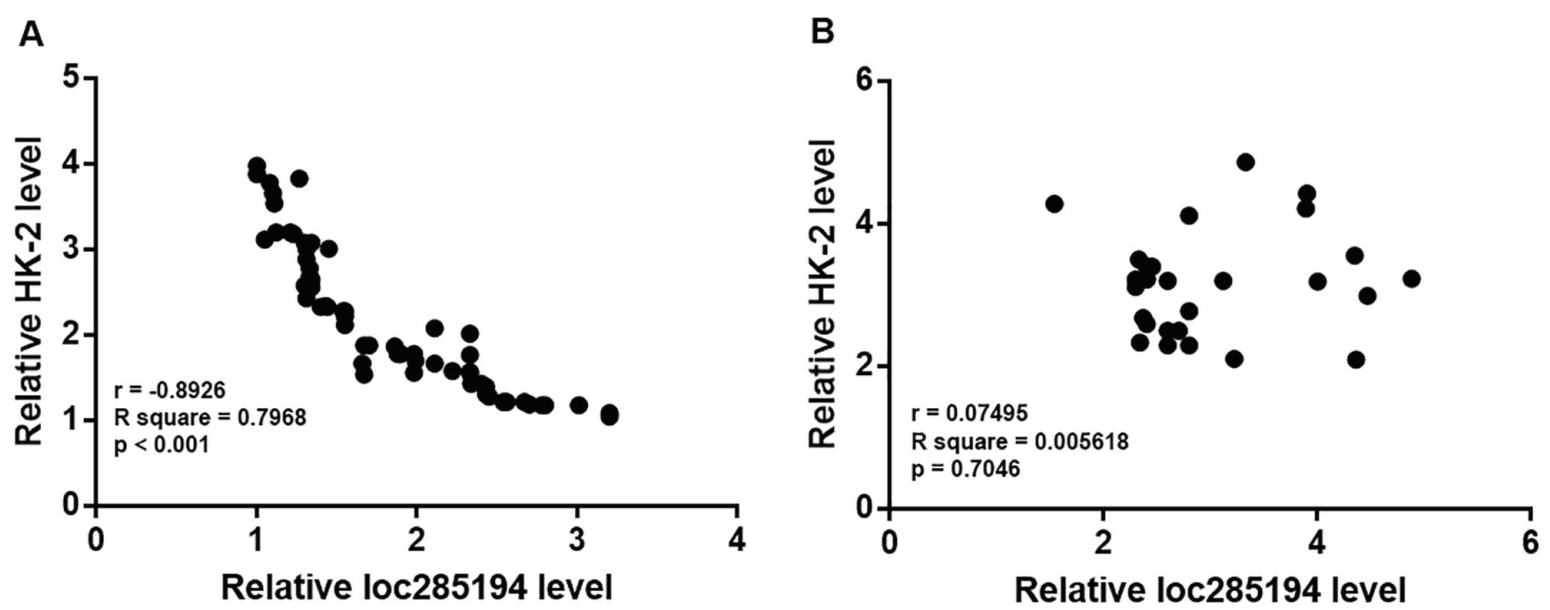

Figure 3. Expression of loc285194 and HK-2 mRNA is inversely correlated in LSCC patients, but not in healthy controls. Pearson correlation coefficient revealed a significant inverse correlation between the expression of loc285194 and HK-2 mRNA in (A) patients with LSCC, but not in (B) healthy controls. LSCC, laryngeal squamous cell carcinoma; HK-2, hexokinase 2.

RT-qPCR and western blot analysis. As shown in Fig. 4A, compared with the control cells and negative control cells, loc285194 overexpression significantly inhibited the expression of HK-2 in the human LSCC UM-SCC-17A cell line, but not in the normal HuLa-PC larynx cell line $(\mathrm{P}<0.05)$. In contrast, HK-2 overexpression demonstrated no significant effects on loc285194 expression in the UM-SCC-17A and HuLa-PC cell lines (Fig. 4B).
Loc285194 overexpression inhibits HK-2 expression and LSCC cell proliferation. A CCK-8 assay was performed to detect cell proliferation following loc285194 and HK-2 overexpression. As shown in Fig. 5, loc285194 overexpression led to significantly inhibited HK-2 expression and LSCC cell proliferation compared with the control and negative control groups $(\mathrm{P}<0.05)$. In addition, HK-2 overexpression attenuated the inhibitory effects of loc285194 overexpression 
A
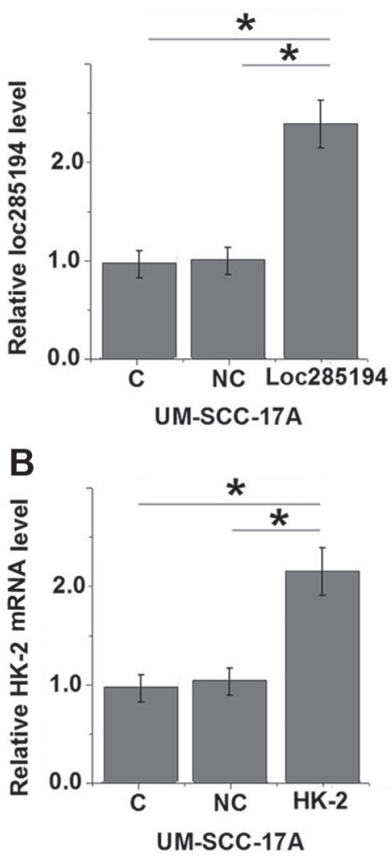
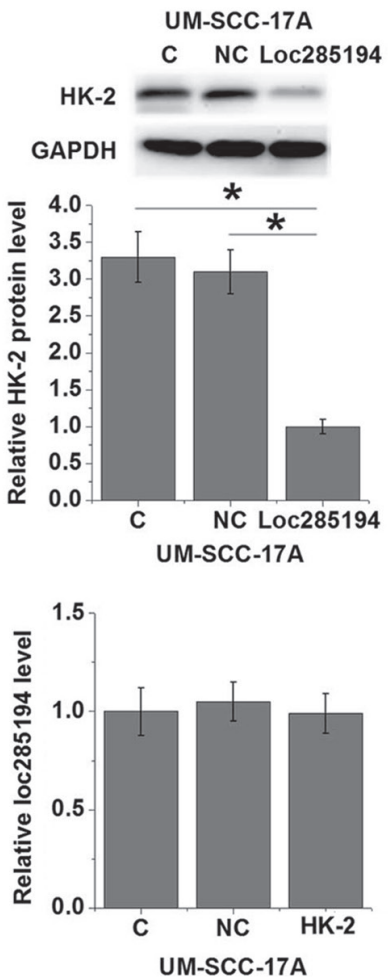
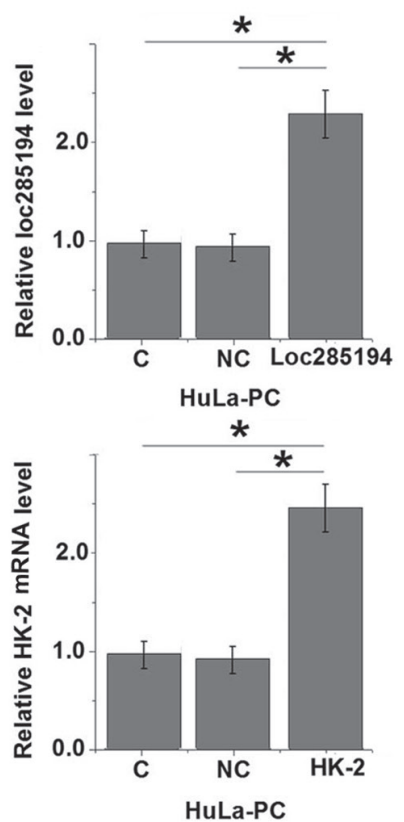

HuLa-PC

C NC Loc285194
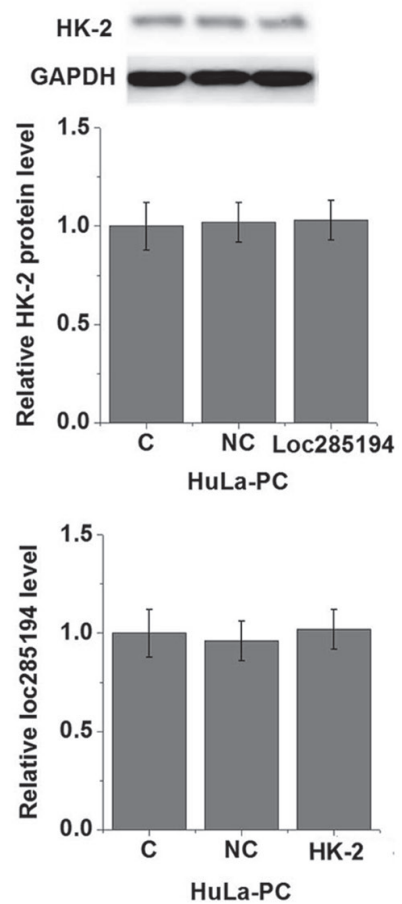

Figure 4. Loc285194 is an upstream activator of HK-2 in LSCC cells. The data demonstrates (A) the effects of loc285194 overexpression on HK-2 expression and (B) the effects of HK-2 overexpression on loc285194 expression. ${ }^{*} \mathrm{P}<0.05$. LSCC, laryngeal squamous cell carcinoma; HK-2, hexokinase 2; C, control; $\mathrm{NC}$, negative control.
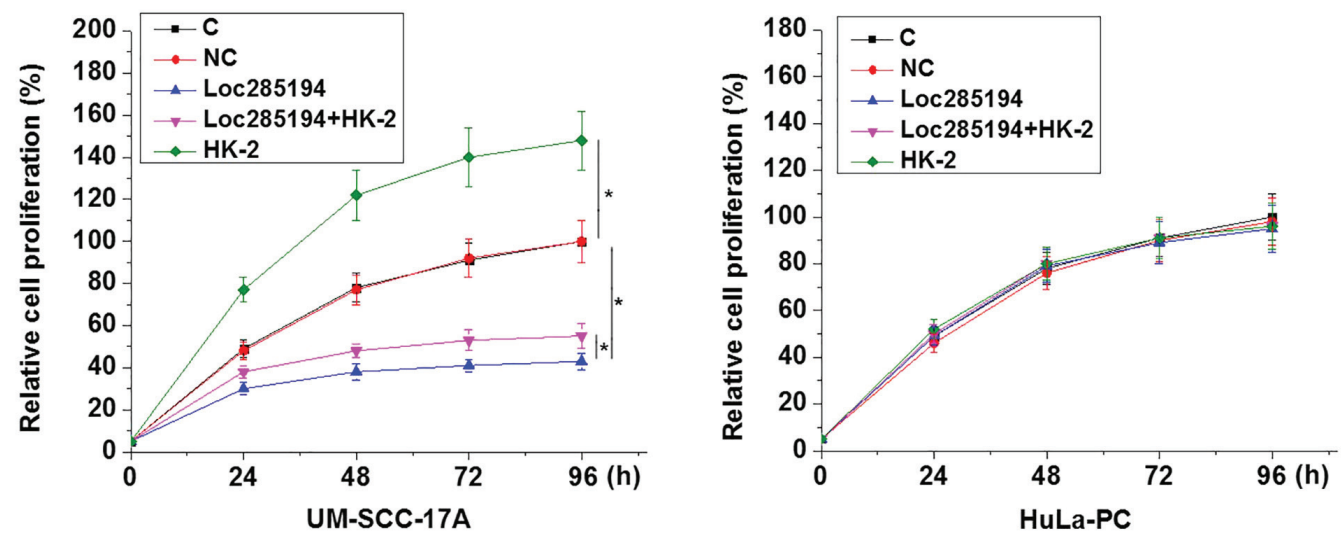

Figure 5. Loc285194 overexpression inhibits and HK-2 overexpression promotes LSCC cell proliferation ${ }^{*} \mathrm{P}<0.05$. The experiment was performed six times. LSCC, laryngeal squamous cell carcinoma; HK-2, hexokinase 2; C, control; NC, negative control.

on LSCC cell proliferation $(\mathrm{P}<0.05)$. Loc285194 and HK-2 overexpression showed no significant effects on the proliferation of normal HuLa-PC cells.

\section{Discussion}

One of the key findings of the present study demonstrated that loc285194, a well-studied tumor suppressor lncRNA in several types of human malignancies, may also participate in the regulation of tumor growth, but not tumor metastasis in patients with LSCC. The action of loc285194 in LSCC is likely to be achieved though interactions with HK-2.

LncRNA loc285194 is a tumor suppressor gene that is downregulated in several types of cancer, including esophageal squamous cell carcinoma (14), pancreatic ductal adenocarcinoma (15) and colorectal cancer (16). In the present study, significantly downregulated expression of lncRNA loc285194 was observed in patients with LSCC comparing with healthy controls. The downregulation of lncRNA loc285194 effectively distinguished patients with LSCC from healthy controls, indicating the potential role of lncRNA loc285194 as a tumor suppressor in LSCC.

Accelerated glucose metabolism distinguishes cancer cells from normal cells (17). HK-2 is a key player in glycolysis and is usually upregulated in the development of cancer (18). In a study on LSCC, Chen et al (9) reported that HK-2 is overexpressed in patients with LSCC and the overexpression of HK-2 promotes tumor growth. Consistent with this 
study, the results of the current study reported that HK-2 was significantly upregulated in patients with LSCC compared with in healthy controls and HK-2 overexpression significantly promoted the proliferation of LSCC cells cultured in vitro. In addition, the overexpression of HK-2 effectively distinguished patients with LSCC from healthy controls.

In the present study, IncRNA loc285194 expression was demonstrated to be significantly associated with tumor size, but not the existing of tumor metastasis, indicating the involvement of lncRNA loc285194 in the regulation of tumor growth. It has been reported that the expression of HK-2 can be regulated by certain IncRNAs $(11,19)$. The results of the current study suggest that lncRNA loc285194 serves as an upstream inhibitor of HK-2 in LSCC. This conclusion may be made based on following observations: i) lncRNA loc285194 overexpression led to a significantly upregulation of $\mathrm{HK}-2$ expression in LSCC cells; ii) HK-2 overexpression showed no significant effects on lncRNA loc285194 expression; iii) the overexpression of $\mathrm{HK}-2$ significantly attenuated the inhibitory effects of lncRNA loc285194 on LSCC cell proliferation. However, the regulatory role of lncRNA loc285194 on HK-2 is unlikely to be direct due to the lack of significant correlation between lncRNA loc285194 on HK-2 in healthy controls and the absence of inhibitory effects of loc285194 expression on HK-2 expression.

Notably, lncRNA loc285194 overexpression demonstrated no significant effects on biological behaviors of normal larynx cells. Therefore, lncRNA loc285194 may serve as a potential therapeutic target for LSCC. However, a previous study has reported that smaller LSCC tumor size is more significantly correlated with an unfavorable prognosis of patients with LSCC (5). Therefore, the correlation between lncRNA loc285194 expression and the prognosis of LSCC patients remains to be further investigated. Overexpression of lncRNA loc285194 also failed to affect the expression of other players in glucose metabolism, such as glucose transporter protein type 1-3 (data not shown). Therefore, lncRNA loc285194 may specifically regulate HK-2. The present study failed to perform HK-2 and loc285194 knockdown assays due to the unsatisfactory knockdown rate. Future studies intend to perform knockdown assays.

In conclusion, the results of the current study demonstrated that lncRNA loc285194 is significantly downregulated in patients with LSCC. LncRNA loc285194 may participate in the regulation of tumor growth in LSCC through the inhibition of HK-2 expression.

\section{Acknowledgements}

Not applicable.

\section{Funding}

Not applicable.

\section{Availability of data and materials}

The datasets used and/or analyzed during the current study are available from the corresponding author on reasonable request.

\section{Authors' contributions}

YZ guaranteed the integrity of the entire study. YZ, YG and $\mathrm{ZW}$ provided the study concepts. YG and ZW were responsible for the study design, the definition of intellectual content, and for literature research. YG, ZW and JT were responsible for clinical and experimental studies. JT was responsible for data acquisition. YG and $\mathrm{ZW}$ were responsible for data analysis, statistical analysis, manuscript preparation, manuscript editing and manuscript review.

\section{Ethics approval and consent to participate}

This study got approval from the ethics committee of the Affiliated Second Hospital Xinjiang Medical University. All participants signed informed consent.

\section{Patient consent for publication}

Not applicable.

\section{Competing interests}

The authors declare that they have no competing interests.

\section{References}

1. Sulciner ML, Serhan CN, Gilligan MM, Mudge DK, Chang J, Gartung A, Lehner KA, Bielenberg DR, Schmidt B, Dalli J, et al: Resolvins suppress tumor growth and enhance cancer therapy. J Exp Med 215: 115-140, 2018.

2. Jemal A, Bray F, Center MM, Ferlay J, Ward E and Forman D: Global cancer statistics. CA Cancer J Clin 61: 69-90, 2011.

3. Ragin CC and Taioli E: Survival of squamous cell carcinoma of the head and neck in relation to human papillomavirus infection: Review and meta-analysis. Int J Cancer 121: 1813-1820, 2007.

4. Zhang SY, Lu ZM, Luo XN, Chen LS, Ge PJ, Song XH, Chen SH and $\mathrm{Wu}$ YL: Retrospective analysis of prognostic factors in 205 patients with laryngeal squamous cell carcinoma who underwent surgical treatment. PLoS One 8: e60157, 2013.

5. Markou K, Goudakos J, Triaridis S, Konstantinidis J, Vital V and Nikolaou A: The role of tumor size and patient's age as prognostic factors in laryngeal cancer. Hippokratia 15: 75-80, 2011.

6. Moreno F, Vega M and Herrero P: The nuclear Hexokinase 2 acts as a glucose sensor in Saccharomyces cerevisiae. J Biol Chem 291: 16478, 2016.

7. Anderson M, Marayati R, Moffitt R and Yeh JJ: Hexokinase 2 promotes tumor growth and metastasis by regulating lactate production in pancreatic cancer. Oncotarget 8: 56081-56094, 2017.

8. Wang L, Xiong H, Wu F, Zhang Y, Wang J, Zhao L, Guo X, Chang LJ, Zhang Y, You MJ, et al: Hexokinase 2-mediated Warburg effect is required for PTEN-and p53-deficiency-driven prostate cancer growth. Cell Rep 8: 1461-1474, 2014.

9. Chen J, Zhang S, Li Y, Tang Z and Kong W: Hexokinase 2 overexpression promotes the proliferation and survival of laryngeal squamous cell carcinoma. Tumour Biol 35: 3743-3753, 2014.

10. Mathupala SP, Ko YH and Pedersen PL: Hexokinase-2 bound to mitochondria: Cancer's stygian link to the 'Warburg Effect' and a pivotal target for effective therapy. Semin Cancer Biol 19: 17-24, 2009.

11. Li Z, Li X, Wu S, Xue M and Chen W: Long non-coding RNA UCA1 promotes glycolysis by upregulating hexokinase 2 through the mTOR-STAT3/microRNA143 pathway. Cancer Sci 105: 951-955, 2014.

12. Liu Q, Huang J, Zhou N, Zhang Z, Zhang A, Lu Z, Wu F and Mo YY: LncRNA loc285194 is a p53-regulated tumor suppressor. Nucleic Acids Res 41: 4976-4987, 2013.

13. Livak KJ and Schmittgen TD: Analysis of relative gene expression data using real-time quantitative PCR and the 2(-Delta Delta C(T)) method. Methods 25: 402-408, 2001. 
14. Tong YS, Zhou XL, Wang XW, Wu QQ, Yang TX, Lv J, Yang JS, Zhu B and Cao XF: Association of decreased expression of long non-coding RNA LOC285194 with chemoradiotherapy resistance and poor prognosis in esophageal squamous cell carcinoma. J Transl Med 12: 233, 2014

15. Ding YC, Yu W, Ma C, Wang Q, Huang CS and Huang T: Expression of long non-coding RNA LOC285194 and its prognostic significance in human pancreatic ductal adenocarcinoma. Int J Clin Exp Pathol 7: 8065-8070, 2014.

16. Qi P, Xu MD, Ni SJ, Huang D, Wei P, Tan C, Zhou XY and Du X: Low expression of LOC285194 is associated with poor prognosis in colorectal cancer. J Transl Med 11: 122, 2013.

17. Shaw RJ: Glucose metabolism and cancer. Curr Opin Cell Biol 18: 598-608, 2006.
18. Wolf A, Agnihotri S, Micallef J, Mukherjee J, Sabha N, Cairns R, Hawkins C and Guha A: Hexokinase 2 is a key mediator of aerobic glycolysis and promotes tumor growth in human glioblastoma multiforme. J Exp Med 208: 313-326, 2011.

19. Zhang Y, Liu Y and Xu X: Knockdown of LncRNA-UCA1 suppresses chemoresistance of pediatric AML by inhibiting glycolysis through the microRNA-125a/hexokinase 2 pathway. J Cell Biochem 119: 6296-6308, 2018.

cc (i) (9) This work is licensed under a Creative Commons Attribution-NonCommercial-NoDerivatives 4.0 International (CC BY-NC-ND 4.0) License. 